\title{
Estrategias de comercialización que inciden en la rentabilidad de los productores de arroz en el distrito de Bagua Grande, provincia de Utcubamba, región Amazonas, 2019
}

\section{Marketing strategies that incident on the profitability of rice producers in the district of Bagua Grande, Utcubamba Province, Amazonas region, 2019}

\section{RESUMEN}

El presente trabajo de investigación: Estrategias de comercialización que inciden en la rentabilidad de los productores de arroz en el distrito de Bagua Grande, provincia de Utcubamba, región Amazonas, 2019; tiene el problema principal: ¿De qué manera las estrategias de comercialización incidirán en la rentabilidad de los productores de arroz en el distrito de Bagua Grande, provincia de Utcubamba, región Amazonas, 2019? Su objetivo general: Determinar qué estrategias de comercialización inciden en la rentabilidad de los productores de arroz en el distrito de Bagua Grande, provincia de Utcubamba, región Amazonas, 2019. La investigación es del tipo descriptiva de diseño no experimental. Cómo resultado principal los productores de arroz obtienen en promedio $\mathrm{S} / 450$ al mes por la producción de arroz por hectárea vendida a precios de chacra durante los 5 meses que dura una campaña. Las conclusiones son: actualmente no están asociados en su gran mayoría, la tecnología es mixta pero obsoleta, la producción se hace sin programaciones efectivas al carecer de recurso hídrico suficiente, y comercialmente no se encuentran asociados y es el intermediario quién compra el arroz en cáscara y lo traslada hacia los molinos de la costa norte del país obteniendo las mayores utilidades de este proceso productivo. Planteándose tres estrategias de comercialización: Agruparlos como pequeños productores de arroz, realizar alianza estratégica en investigación de una nueva variedad de arroz con la UNTRM, y la propuesta de un estudio de pre inversión para la construcción de mini represas de almacenamiento de agua para riego en Bagua Grande.

Palabras clave: Estrategias, rentabilidad, comercialización y arroz

\begin{abstract}
The present research work: Marketing strategies that affect the profitability of rice producers in the district of Bagua Grande, province of Utcubamba, Amazonas region, 2019; It has the main problem: How will marketing strategies affect the profitability of rice producers in the Bagua Grande district, Utcubamba province, Amazonas region, 2019? Its general objective: To determine which marketing strategies affect the profitability of rice producers in the district of Bagua Grande, province of Utcubamba, Amazonas region, 2019. The research is of the descriptive type of non-experimental design. As a main result, rice producers obtain an average of S / 450 per month for the production of rice per hectare sold at farm prices during the 5 months of a campaign. The conclusions are: currently they are not associated in their great majority, the technology is mixed but obsolete, the production is done without effective programming when lacking sufficient water resources, and commercially they are not associated and it is the intermediary who buys the rice in the shell and moves it to the mills of the north coast of the country obtaining the greatest profits from this production process. Three marketing strategies are proposed: Grouping them as small rice producers, making a strategic alliance in the research of a new rice variety with the UNTRM, and the proposal of a pre-investment study for the construction of mini water storage dams for irrigation in Bagua Grande.
\end{abstract}

Keywords: Strategies, profitability, commercialization and rice.

'Bachiller en Economía. Universidad Nacional Toribio Rodríguez de Mendoza de Amazonas. Correo electrónico: nataliayarihuaman@hotmail.com.

${ }^{2}$ Docente Auxiliar de la Facultad de Ciencias Económicas y Administrativas de la Universidad Nacional Toribio Rodríguez de Mendoza de Amazonas; Economista, Universidad Nacional de Trujillo; magíster en Ciencias Económicas, Mención: Proyectos de Inversión; Universidad Nacional de Piura. Correo electrónico: raul.poemape@untrm.edu.pe. 


\section{INTRODUCCIÓN}

Según De Bernardi, (2016), mencionan que el arroz es una especie perteneciente a la familia de las gramíneas, cuya semilla debidamente procesada constituye la base alimentaria de más de un tercio de la población mundial. Las bondades nutricionales del arroz han trascendido por su importancia, dado que esta gramínea es la que mayor aporte calórico brinda de todos los cereales. El almidón es el componente principal del grano de arroz, y se encuentra en un rango que va del $70 \%$ al $80 \%$. El arroz blanco pulido, ya desprovisto de su cáscara y su salvado, suele tener menos fibra dietética que otros cereales y por lo tanto alcanza un nivel de digestibilidad superior. Actualmente se cultiva en 113 países con un nivel de producción según lo estimado por el departamento de Agricultura de Estados Unidos para la campaña 2016-2017, de 481, 5 millones de toneladas. Los precios a nivel global fueron oscilantes y terminaron por registrar una importante caída debido a las abundantes disponibilidades de materia prima. La contracción de los precios fue significativa en Pakistán y también en Tailandia. A pesar de la ligera reactivación de la demanda, ya sea por productividad y stocks, no se vislumbran elementos que permitan hacer suponer que los precios podrían aumentar en lo inmediato o para el primer semestre del 2017.

Según el Ministerio de Agricultura y Riego (2017), el distrito de Bagua Grande es eminentemente arrocero, como parte de la provincia de Utcubamba. La región Amazonas representa el 10\% de participación como región productora de arroz en cáscara en el año 2016, con rendimientos de $7.4 \mathrm{Tm} / \mathrm{Ha}$, por debajo del promedio nacional de $7.5 \mathrm{Tm} / \mathrm{Ha}$. En relación al precio del productor en nuestra región es de $\mathrm{S} / 1.03$ por kilo, precio en chacra, muy por debajo del promedio nacional que alcanza $\mathrm{S} / 1.15$ por kilo.

Luego, este arroz cosechado debe ser transformado en el producto final que llega al consumidor final hasta S/. 3.00 por kilo en el propio Bagua Grande, a pesar de que la región Amazonas cuenta con 55 molinos al año 2016.

\section{MATERIAL Y MÉTODOS}

Se realizó una entrevista estructurada a los productores arroceros in situ en marzo del presente año 2019, teniendo los siguientes resultados.

3.1 Determinación de la capacidad directiva, tecnológica, productiva y comercial de los productores de arroz en el distrito de Bagua Grande.

Tabla $\mathrm{N}^{\circ} 01$. Ubicación de su parcela de arroz

Los resultados indican que, del total de entrevistados, el $36.7 \%$ tiene su parcela de arroz en el caserío El Pintor, seguido del $8.3 \%$ en Morerilla Baja, otro $8.3 \%$ en el sector Abad, un $6.7 \%$ en Nuñay Temple y otro 6.7\% en Morropón -Winguío.

\begin{tabular}{|l|c|c|}
\multirow{2}{*}{\multicolumn{1}{|c|}{ Ubicación }} & \multicolumn{2}{c|}{ Total } \\
\cline { 2 - 3 } & Datos & Porcentaje \\
\hline El Pintor & 22 & $36.7 \%$ \\
\hline Morerilla Baja & 5 & $8.3 \%$ \\
\hline Abad & 5 & $8.3 \%$ \\
\hline Ñuñay Temple & 4 & $6.7 \%$ \\
\hline Morropón - Winguío & 4 & $6.7 \%$ \\
\hline Santa Elena & 3 & $5.0 \%$ \\
\hline Morerilla Alta & 3 & $5.0 \%$ \\
\hline Huarango & 3 & $5.0 \%$ \\
\hline La Victoria & 3 & $5.0 \%$ \\
\hline La Versalla & 2 & $3.3 \%$ \\
\hline Bocana & 2 & $3.3 \%$ \\
\hline Quebrada Seca & 1 & $1.7 \%$ \\
\hline Jahuanga & 1 & $1.7 \%$ \\
\hline San Antonio & 1 & $1.7 \%$ \\
\hline Quebrada Honda & 1 & $1.7 \%$ \\
\hline Total & 60 & $\mathbf{1 0 0 . 0} \%$ \\
\hline Fuente: Entrevista a productores arroceros, marzo 2019
\end{tabular}

\section{En cuanto a capacidad o gestión directiva}

Tabla $\mathbf{N}^{\circ} 02$. ¿Qué superficie de terreno cultiva usted en hectáreas?

\begin{tabular}{|l|c|c|}
\hline \multicolumn{1}{|c|}{$\mathbf{N}^{\circ}$ de hectáreas cultivadas } & Cantidad & $\%$ \\
\hline Menos de 1 & 0 & $0.0 \%$ \\
\hline de 1 hasta 5 & 30 & $50.0 \%$ \\
\hline más de 5 hasta 10 & 16 & $26.7 \%$ \\
\hline más de 10 & 14 & $23.3 \%$ \\
\hline Total & 60 & $100.0 \%$ \\
\hline Fuente: Entrevista a productores arroceros, marzo 2019
\end{tabular}

De los entrevistados, el 50\% cultiva entre 1 hasta 5 hectáreas, el $26.7 \%$ de 5 a 10 hectáreas y el $23.3 \%$ cultiva más de 10 hectáreas.

Tabla $\mathbf{N}^{\circ}$ 03. Sobre los terrenos de sembrío, estos son:

\begin{tabular}{|l|c|c|}
\multicolumn{1}{|c|}{ Tenencia de su terreno } & Cantidad & $\%$ \\
\hline Propios & 29 & $48.3 \%$ \\
\hline alquilados & 28 & $46.7 \%$ \\
\hline otro tipo: herencia familiar & 3 & $5.0 \%$ \\
\hline Total & 60 & $100.0 \%$ \\
\hline
\end{tabular}

Fuente: Entrevista a productores arroceros, marzo 2019

Preguntando sobre la tenencia de sus terrenos, el $48.3 \%$ indica que son propios, el $46.7 \%$ son alquilados y un $5 \%$ es del tipo herencia familiar.

Tabla $\mathbf{N}^{\circ} \mathbf{0 4}$. ¿Hace cuánto tiempo en años se dedica Ud. a la producción de arroz?

\begin{tabular}{|l|c|c|}
\multicolumn{1}{|c|}{ Hace cuanto tiempo es productor } & Cantidad & $\%$ \\
\hline Menos de 5 años & 6 & $10.0 \%$ \\
\hline Más de 5 y menor a 10 años & 12 & $20.0 \%$ \\
\hline Más de 10 y menor a 20 años & 22 & $36.7 \%$ \\
\hline Más de 20 y menor a 30 años & 16 & $26.7 \%$ \\
\hline Más de 30 años & 4 & $6.7 \%$ \\
\hline Total & 60 & $100.0 \%$ \\
\hline Fuente: Entrevista a productores arroceros, marzo 2019
\end{tabular}

En cuanto al tiempo en años que se dedica a esta 
actividad, el $36.7 \%$ lo hace en más de 10 años y menos de 20 años, el $26.7 \%$ en más de 20 años y menos de 30 años, y el $20 \%$ entre 5 años y 10 años.

Tabla $\mathbf{N}^{\circ} 05$ : ¿En qué meses realiza esta producción?

\begin{tabular}{|l|c|c|}
\hline \multicolumn{1}{|c|}{ Meses de siembra y cosecha } & Cantidad & $\%$ \\
\hline Siembra: & & \\
\hline Diciembre a febrero & 47 & $39.2 \%$ \\
\hline febrero & 11 & $9.2 \%$ \\
\hline octubre - noviembre & 2 & $1.7 \%$ \\
\hline Cosecha: & 47 & \\
\hline Abril a junio & 11 & $39.2 \%$ \\
\hline junio & 2 & $1.7 \%$ \\
\hline marzo - abril & $\mathbf{1 2 0}$ & $100.0 \%$ \\
\hline Total & &
\end{tabular}

Casi un $40 \%$ siembra entre diciembre a febrero y cosecha entre abril a junio.

Tabla $\mathbf{N}^{\circ} 06$. ¿Planifica Ud. la producción de arroz?

\begin{tabular}{l|c|c|}
\hline \multicolumn{1}{|c|}{ Manera de planificar } & $\mathbf{1 1}$ & $\%$ \\
\hline (No): & 47 & $78.3 \%$ \\
\hline (Sí): Por campañas de riego & 10 & $16.7 \%$ \\
\hline (Si): Cuando aparecen las lluvias & 3 & $5.0 \%$ \\
\hline Total & 60 & $100.0 \%$ \\
\hline Fuente: Entrevista a productores arroceros, marzo 2019
\end{tabular}

Un dato relevante para la investigación, es un 78.3\% de los productores entrevistados no realiza planificación alguna de su producción de arroz. Sólo el $16.7 \%$ planifica de acuerdo a las campañas de riego y un $5 \%$ cuando aparecen las lluvias.

\section{En cuanto a capacidad o gestión tecnológica}

Tabla $\mathbf{N}^{\circ}$ 07. ¿Qué tipo de siembra realiza normalmente

\begin{tabular}{|l|c|c|}
\hline \multicolumn{1}{|c|}{ Tipo de siembra } & Cantidad & $\%$ \\
\hline Mecanizada & 39 & $65.0 \%$ \\
\hline manual & 3 & $5.0 \%$ \\
\hline mixta & 18 & $30.0 \%$ \\
\hline Total & 60 & $100.0 \%$ \\
\hline
\end{tabular}

Fuente: Entrevista a productores arroceros, marzo 2019

El 65\% respondió el tipo de siembra es mecanizada, el 30\% es mixta y sólo un 5\% es manual.

Tabla $\mathbf{N}^{\circ} \mathbf{0 8}$. ¿Qué variedad de semilla utiliza para esta producción?

\begin{tabular}{|l|c|c|}
\hline \multicolumn{1}{|c|}{ Variedad de semilla de arroz } & Cantidad & $\%$ \\
\hline Ferón & 17 & $28.3 \%$ \\
\hline Esperanza & 40 & $66.7 \%$ \\
\hline Capirona & 3 & $5.0 \%$ \\
\hline Total & 60 & $100.0 \%$ \\
\hline
\end{tabular}

Fuente: Entrevista a productores arroceros, marzo 2019

Autor: Natalia G. Yarihuaman Sánchez

Mayormente usan en el distrito de Bagua Grande la variedad de Esperanza en un $66.7 \%$, el $28.3 \%$ usa ferón y el $5 \%$ usa capirona.

Tabla $\mathbf{N}^{\circ} 09$. ¿Utiliza semilla certificada para su cultivo de arroz? ¿ ¿Dónde las adquiere?

\begin{tabular}{|l|c|c|}
\hline \multicolumn{1}{|c|}{ Usa semilla certificada } & Cantidad & $\%$ \\
\hline No & 10 & $16.7 \%$ \\
\hline Sí, en Bagua Grande: Agrícola El Sembrador & 8 & $13.3 \%$ \\
\hline Si en Bagua Grande: Agrícola del Norte & 5 & $8.3 \%$ \\
\hline Sí en Bagua Grande: Agrícola El Campesino & 3 & $5.0 \%$ \\
\hline Sí en Bagua Grande: Agronegocios Torres & 5 & $8.3 \%$ \\
\hline Sí en Bagua Grande: El Potrero & 4 & $6.7 \%$ \\
\hline Indistintamente en Bagua Grande & 22 & $36.7 \%$ \\
\hline En otra ciudad: Jaén (El Potrero) & 3 & $5.0 \%$ \\
\hline Total & 60 & $100.0 \%$ \\
\hline Fuente: Entrevista productores arroceros, marzo 2019
\end{tabular}

Fuente: Entrevista a productores arroceros, marzo 2019

El 83.3\% sí utiliza semilla certificada adquiriéndolas mayormente en las tiendas especializadas de la ciudad de Bagua Grande y el 16.7\% no utiliza semilla certificada.

Tabla $\mathbf{N}^{\circ} \mathbf{1 0}$. ¿De dónde proviene el agua que utiliza en su siembra? Indicar nombre del canal de regadío u otro.

\begin{tabular}{|l|c|c|}
\hline \multicolumn{1}{|c|}{ De donde proviene el agua } & Cantidad & $\%$ \\
\hline Quebrada El Pintor & 31 & $51.7 \%$ \\
\hline Canal de Morerilla & 8 & $13.3 \%$ \\
\hline Quebrada Ñuñya Temple & 5 & $8.3 \%$ \\
\hline Canal Abad & 5 & $8.3 \%$ \\
\hline Otro canal sin nombre & 9 & $15.0 \%$ \\
\hline Río Utcubamba & 2 & $3.3 \%$ \\
\hline Total & 60 & $100.0 \%$ \\
\hline Fuente: Entrevista a productores arroceros, marzo 2019
\end{tabular}

El 96.7\% usa agua de quebrada o de canales en sus zonas de siembra, y sólo el $3.3 \%$ usa agua del río Utcubamba.

Tabla $\mathbf{N}^{\circ}$ 11. ¿Cuánto es el pago por el servicio de agua para riego y cada que tiempo lo realiza?

\begin{tabular}{|l|c|c|}
\hline \multicolumn{1}{|c|}{ Monto del pago por el agua } & Cantidad & $\%$ \\
\hline S/ 8.00 mensual por hectárea & 2 & $3.3 \%$ \\
\hline S/ 10.00 mensual por hectárea & 36 & $60.0 \%$ \\
\hline S/ 12.00 mensual por hectárea & 11 & $18.3 \%$ \\
\hline S/ 15.00 mensual por hectárea & 11 & $18.3 \%$ \\
\hline Total & 60 & $100.0 \%$ \\
\hline Fuente: Entrevista a productores arroceros, marzo 2019
\end{tabular}

El $60 \%$ indica pagar S/ 10.00 por hectárea, el 18.3\% paga $\mathrm{S} / 12.00$ y otro $18.3 \%$ paga $\mathrm{S} / 15.00$.

Tabla $\mathbf{N}^{\circ} 12$ ¿ ¿Qué tipo de control de malezas utiliza mayormente?

\begin{tabular}{l|c|c|}
\hline \multicolumn{1}{c|}{ Control de malezas } & Cantidad & $\%$ \\
\hline Control manual & 0 & $0.0 \%$ \\
\hline mecanizado & 0 & $0.0 \%$ \\
\hline Control químico & 60 & $100.0 \%$ \\
\hline Total & 60 & $100.0 \%$ \\
\hline
\end{tabular}

Fuente: Entrevista a productores arroceros, marzo 2019

Sobre la manera de control de malezas, el 100\% usa en control químico.

Tabla $N^{\circ}$ 13. ¿Sus cultivos se han afectado por alguna enfermedad o plagas?

\begin{tabular}{|l|c|c|}
\hline \multicolumn{1}{|c|}{ Control de malezas } & Cantidad & $\%$ \\
\hline No & 13 & $21.7 \%$ \\
\hline Sí: Sogatas y gusanos & 1 & $1.7 \%$ \\
\hline Sí: sólo malezas & 3 & $5.0 \%$ \\
\hline Sí, picularia y manchas & 12 & $20.0 \%$ \\
\hline Sí, bacterias y chinches & 31 & $51.7 \%$ \\
\hline Total & 60 & $100.0 \%$ \\
\hline Fuente: Entrevista a productores arroceros, marzo 2019
\end{tabular}


El $21.7 \%$ indica no se han afectado sus cultivos por enfermedades o plagas, el $51.7 \%$ manifiesta que sí por bacterias y chinches y el $20 \%$ manifiesta que sí por la picularia y manchas.

Tabla $\mathbf{N}^{\circ}$ 14. ¿Cómo le dio solución a esta plaga o enfermedad?

\begin{tabular}{|l|c|c|}
\hline \multicolumn{1}{c|}{ Solución a plagas o enfermedades } & Cantidad & $\%$ \\
\hline Fumigación (herbicidas, insecticidas y fungicidas) & 27 & $45.0 \%$ \\
\hline Control químico & 17 & $28.3 \%$ \\
\hline Ningún control & 16 & $26.7 \%$ \\
\hline Total & 60 & $100.0 \%$ \\
\hline Fuente: Entrevista a productores arroceros, marzo 2019 \\
\hline
\end{tabular}

Y el 45\% le dio solución con fumigación, el 28.3\% con control químico y un $26.7 \%$ manifestó que no le dio ningún control.

\section{En cuanto a capacidad o gestión productiva}

Tabla $\mathbf{N}^{\circ}$ 15. ¿Cuál es rendimiento por hectárea que obtiene durante un ciclo del cultivo?

\begin{tabular}{|l|c|c|} 
Rendimiento promedio & Cantidad & $\%$ \\
\hline $8,000 \mathrm{Kg}$ & 8 & $13.3 \%$ \\
\hline $7,900 \mathrm{Kg}$ & 4 & $6.7 \%$ \\
\hline $7,800 \mathrm{Kg}$ & 16 & $26.7 \%$ \\
\hline $7,600 \mathrm{Kg}$ & 20 & $33.3 \%$ \\
\hline $7,200 \mathrm{Kg}$ & 11 & $18.3 \%$ \\
\hline $6,800 \mathrm{Kg}$ & 1 & $1.7 \%$ \\
\hline Total & 60 & $100.0 \%$ \\
\hline Fuente: Entrevista a productores arroceros, marzo 2019
\end{tabular}

El 33.3\% obtiene rendimientos promedios de 7,600 $\mathrm{Kg}$ por hectárea, el $26.7 \%$ obtiene $7,800 \mathrm{~kg} / \mathrm{ha}$, el $18.3 \%$ obtiene $7,200 \mathrm{~kg} / \mathrm{ha}$, y sólo el $13.3 \%$ indica obtener $8,000 \mathrm{~kg} / \mathrm{ha}$.

Tabla $\mathbf{N}^{\circ}$ 16. ¿Tiene pérdidas en esta producción de arroz?

\begin{tabular}{|c|c|c|}
\hline Tiene perdidas & Cantidad & $\%$ \\
\hline No & 56 & $93.3 \%$ \\
\hline \multicolumn{3}{|l|}{ Sí } \\
\hline S/ 1,000 por Hás & 2 & $3.3 \%$ \\
\hline Baja el precio & 2 & $3.3 \%$ \\
\hline Total & 60 & $100.0 \%$ \\
\hline
\end{tabular}

Fuente: Entrevista a productores arroceros, marzo 2019

Un $93.3 \%$ indica no tener pérdidas con la producción actual de arroz; pero el $6.6 \%$ manifiesta tener pérdidas por el bajo precio de comercialización o $\mathrm{S} /$ 1,000.00 por hectárea en promedio.

Tabla $\mathbf{N}^{\circ}$ 17. ¿Cuál es el periodo de almacenamiento de su producto antes de la comercialización?

\begin{tabular}{|l|c|c|}
\hline \multicolumn{1}{|c|}{ Tiempo de almacenamiento } & Cantidad & $\%$ \\
\hline Menor a 1 día & 16 & $26.7 \%$ \\
\hline de 1 a 7 días & 44 & $73.3 \%$ \\
\hline de 8 a 15 días & 0 & $0.0 \%$ \\
\hline más de 15 días & 0 & $0.0 \%$ \\
\hline Total & 60 & $100.0 \%$ \\
\hline Fuente Entrevista a productores arroceros, marzo 2019
\end{tabular}

Normalmente almacena el arroz entre 1 a 7 días en un $73.3 \%$, y el $26.7 \%$ indica almacenarlo menos de un día, es decir lo comercializa rápidamente en chacra.
Tabla $N^{\circ}$ 18. Generalmente ¿Cuál es la modalidad de comercialización de su arroz?

\begin{tabular}{l|c|c|}
\multicolumn{1}{|c|}{ Manera de comercialización } & Cantidad & $\%$ \\
\hline En cáscara & 60 & $100.0 \%$ \\
\hline en grano & 0 & $0.0 \%$ \\
\hline Total & 60 & $100.0 \%$ \\
\hline
\end{tabular}

Fuente: Entrevista a productores arroceros, marzo 2019

La totalidad de productores entrevistados indica que comercializa su arroz en cáscara.

Tabla $N^{\circ} 19$. ¿Cuál es la modalidad de la venta de su producción de arroz?

\begin{tabular}{l|c|c|}
\multicolumn{1}{c|}{ Modalidad de venta } & Cantidad & $\%$ \\
\hline Venta en chacra & 46 & $76.7 \%$ \\
\hline la lleva al molino cercano & 2 & $3.3 \%$ \\
\hline al intermediario & 1 & $1.7 \%$ \\
\hline al mayorista & 1 & $1.7 \%$ \\
\hline lo traslada al molino a Chiclayo & 8 & $13.3 \%$ \\
\hline al por menor & 0 & $0.0 \%$ \\
\hline a la tienda comercial o empresario & 2 & $3.3 \%$ \\
que le dio crédito & 0 & $0.0 \%$ \\
\hline otro & 60 & $100.0 \%$ \\
\hline Total & \multicolumn{2}{|c|}{}
\end{tabular}

Fuente: Entrevista a productores arroceros, marzo 2019

El 76.7\% manifiesta vender su producción en chacra, el $13.3 \%$ la lleva hasta los molinos en la ciudad de Chiclayo, el 3.3\% la lleva al molino más cercano en Bagua Grande y otro 3.3\% lo vende a la tienda comercial que le dio crédito para esta producción.

Tabla $\mathbf{N}^{\circ} 20$. ¿Cuál es el precio del saco de arroz mediante esta venta?

\begin{tabular}{l|c|c|}
\multicolumn{1}{|c|}{ Precio del saco de arroz } & Cantidad & $\%$ \\
\hline Entre S/ 60 y S/ 70 & 7 & $11.7 \%$ \\
\hline Más de S/ 70 hasta S/ 80 & 33 & $55.0 \%$ \\
\hline Más de S/ 80 hasta S/ 90 & 11 & $18.3 \%$ \\
\hline Más de S/ 90 hasta S/ 100 & 9 & $15.0 \%$ \\
\hline Más de S/ 100 hasta S/ 110 & 0 & $0.0 \%$ \\
\hline Total & 60 & $100.0 \%$ \\
\hline Fuente: Entrevista a productores arroceros, marzo 2019
\end{tabular}

El 55\% reciben entre $\mathrm{S} / 70.00$ a S/ 80.00 por un saco de arroz en cáscara, el $18.3 \%$ recibe entre $\mathrm{S} / 80.00$ a $\mathrm{S} / 90.00$ y el $15 \%$ recibe entre S/ 90.00 a S/ 100.00 .

Tabla $\mathbf{N}^{\circ} 21$. ¿Cuántos kilos pesa del saco vendido?

\begin{tabular}{l|c|c|}
\multicolumn{1}{c|}{ Peso del saco de arroz } & Cantidad & $\%$ \\
\hline Entre $70 \mathrm{Kg} \mathrm{y} 80 \mathrm{Kg}$ & 2 & $3.3 \%$ \\
\hline Más de $80 \mathrm{Kg}$ hasta $90 \mathrm{Kg}$ & 9 & $15.0 \%$ \\
\hline Más de $90 \mathrm{Kg}$ hasta $100 \mathrm{Kg}$ & 31 & $51.7 \%$ \\
\hline Más de $100 \mathrm{Kg}$ hasta $110 \mathrm{Kg}$ & 18 & $30.0 \%$ \\
\hline Más de $110 \mathrm{Kg}$ hasta $120 \mathrm{Kg}$ & 0 & $0.0 \%$ \\
\hline Total & 60 & $100.0 \%$ \\
\hline Fuente: Entrevista a productores arroceros, marzo 2019
\end{tabular}

El $51.7 \%$ indica que el saco vendido tiene un peso entre los $90 \mathrm{~kg}$ a $100 \mathrm{~kg}$, y el $30 \%$ tiene entre $100 \mathrm{~kg}$ y $110 \mathrm{~kg}$. 
Tabla $\mathrm{N}^{\circ} 22$. Precio por $\mathrm{Kg}$ de arroz vendido

\begin{tabular}{|l|c|c|}
\multicolumn{1}{|c|}{ Precio por kilo de arroz } & Cantidad & $\%$ \\
\hline Entre S/ 0.70 y S/ 0.80 & 41 & $68.3 \%$ \\
\hline Más de S/ 0.80 hasta S/ 0.90 & 19 & $31.7 \%$ \\
\hline Más de S/ 0.90 hasta S/ 1.00 & 0 & $0.0 \%$ \\
\hline Más de S/ 1.00 hasta S/ 1.10 & 0 & $0.0 \%$ \\
\hline Más de S/ 1.10 hasta S/ 1.20 & 0 & $0.0 \%$ \\
\hline Total & 60 & $100.0 \%$ \\
\hline
\end{tabular}

Fuente: Entrevista a productores arroceros, marzo 2019

Pero el $68.3 \%$ manifiesta recibir entre $\mathrm{S} / 0.70$ a S/ 0.80 por kilo de arroz vendido en chacra y el $31.7 \%$ indica recibir entre $\mathrm{S} / 0.80$ a $\mathrm{S} / 0.90$.

Tabla $\mathbf{N}^{\circ} 23$. ¿Cuál es el monto total que recibe por la venta de arroz por hectárea?

\begin{tabular}{|l|c|c|}
\hline Monto que recibo por su venta & Cantidad & $\%$ \\
\hline Entre S/ 5,000 hasta S/ 6,000 & 32 & $53.3 \%$ \\
\hline Entre S/ 6,000 y S/ 7,000 & 21 & $35.0 \%$ \\
\hline Entre S/ 7,000 y S/ 8,000 & 7 & $11.7 \%$ \\
\hline Entre S/ 8,000 y S/ 9,000 & 0 & $0.0 \%$ \\
\hline Entre S/ 9,000 y S/ 10,000 & 0 & $0.0 \%$ \\
\hline Total & 60 & $100.0 \%$ \\
\hline Fuente: Entrevista a productores arroceros, marzo 2019
\end{tabular}

El 53.3\% manifiesta recibir entre $\mathrm{S} / 5,000.00$ a S/ $6,000.00$ por la venta de su arroz por hectárea, y un $35 \%$ indica recibir entre $\mathrm{S} / 6,000$ a S/ 7,000.00.

\section{En cuanto a capacidad o gestión comercial o marketing}

Tabla $N^{\circ}$ 24. ¿Pertenece Ud. a alguna asociación $u$ organización de productores de arroz?

\begin{tabular}{|l|c|c|}
\hline \multicolumn{1}{|c|}{ Se encuentra asociado } & Cantidad & $\%$ \\
\hline No & 45 & $75.0 \%$ \\
\hline Sí & 0 & $0.0 \%$ \\
\hline Del caserío El Pintor & 10 & $16.7 \%$ \\
\hline Asociación de Bagua Grande & 5 & $8.3 \%$ \\
\hline Total & 60 & $100.0 \%$ \\
\hline
\end{tabular}

Fuente: Entrevista a productores arroceros, marzo 2019

El 75\% manifiesta no pertenecer a alguna asociación u organización, frente a un $25 \%$ que indica sí estar asociado en el caserío El Pintor o en la ciudad de Bagua Grande.

Tabla $\mathbf{N}^{\circ}$ 25. ¿Ha participado en cursos de capacitación sobre producción o comercialización de arroz?

\begin{tabular}{|l|c|c|}
\hline \multicolumn{1}{|c|}{ Se capacitó } & Cantidad & $\%$ \\
\hline No & 56 & $93.3 \%$ \\
\hline Sí & 0 & $0.0 \%$ \\
\hline Tipos de arroz de Molinos y CIA & 2 & $3.3 \%$ \\
\hline Control de maleza y químicos TQC & 2 & $3.3 \%$ \\
\hline Total & 60 & $100.0 \%$ \\
\hline Fuente: Entrevista a productores arroceros, marzo 2019
\end{tabular}

El 93.3\% indicó no haber recibido capacitación alguna, frente al $6.6 \%$ que sí recibió capacitación en tipos de arroz o en control de maleza y químicos.
Tabla $N^{\circ}$ 26. ¿Cuál es el tipo de tecnología que utiliza para su producción de arroz?

\begin{tabular}{|l|c|c|}
\multicolumn{1}{r|}{ Usa tecnología en su producción } & Cantidad & $\%$ \\
\hline No utiliza & 0 & $0.0 \%$ \\
\hline con maquinaria & 60 & $100.0 \%$ \\
\hline otros & 0 & $0.0 \%$ \\
\hline Total & 60 & $100.0 \%$ \\
\hline
\end{tabular}

Fuente: Entrevista a productores arroceros, marzo 2019

El $100 \%$ identifica utilizar maquinaria en el proceso de su producción de arroz.

Tabla $\mathbf{N}^{\circ}$ 27. ¿Sabe Ud. cuánto es el precio por kilo de arroz pilado en Bagua Grande?

\begin{tabular}{|l|c|c|}
\multicolumn{1}{|c|}{ Precio del kilo de arroz pilado } & Cantidad & $\%$ \\
\hline S/. 2.50 & 19 & $31.7 \%$ \\
\hline S/. 2.80 & 8 & $13.3 \%$ \\
\hline S/. 3.00 & 29 & $48.3 \%$ \\
\hline S/. 3.20 & 0 & $0.0 \%$ \\
\hline S/. 3.50 & 4 & $6.7 \%$ \\
\hline Total & 60 & $100.0 \%$ \\
\hline Fuen
\end{tabular}

Respecto a conocer el precio del arroz por kilo en la ciudad de Bagua Grande, indica el $48.3 \%$ estar a S/ 3.00 , el $31.7 \%$ indican un $\mathrm{S} / 2.50$ y el $13.3 \%$ indican $\mathrm{S} / 2.80$ por kilo.

Tabla $\mathbf{N}^{\circ}$ 28. Generalmente el precio del arroz en chacra oscila entre $\mathrm{S} / .1 .00 \mathrm{y} \mathrm{S} / .1 .20 \mathrm{y}$ el precio del arroz pilado al público está S/. 2.80 a S/. 3.00 ¿Puede identificar a que se debe esto?

\begin{tabular}{|l|c|c|}
\hline \multicolumn{1}{|c|}{ Identificación sobre precios } & Cantidad & $\%$ \\
\hline Mayores costos en transportes y el servicio de pilado & 26 & $43.3 \%$ \\
\hline Genera mayor trabajo e inversión & 7 & $11.7 \%$ \\
\hline Presencia de los intermediarios & 13 & $21.7 \%$ \\
\hline El gobierno no apoya al arrocero & 10 & $16.7 \%$ \\
\hline Manejo del precio en los molinos & 4 & $6.7 \%$ \\
\hline Total & 60 & $100.0 \%$ \\
\hline Fuente: Entrevista a productores arroceros, marzo 2019 \\
\hline
\end{tabular}

En cuanto a preguntar sobre la diferenciación notoria del precio del arroz en chacra y el arroz pilado al público, el 43,3\% señalan por mayores costos en transportes para el pilado, el $21.7 \%$ manifiesta por la presencia de intermediarios, el $16.7 \%$ señala que el gobierno no apoya a este sector y un $11.7 \%$ porque les genera mayor trabajo e inversión.

Tabla $\mathbf{N}^{\circ} 29$. ¿Por qué no realiza Ud. directamente el servicio de pilado de su arroz y lo vendería al por menor?

\begin{tabular}{|l|c|c|}
\multicolumn{1}{|c|}{ Porqué no realiza la venta directa de su arroz } & Cantidad & $\%$ \\
\hline Se incrementan sus costos y disminuyen sus ingresos & 2 & $3.3 \%$ \\
\hline Por la demora en pilarlo y venderlo & 14 & $23.3 \%$ \\
\hline Por desconocimiento al ser sólo productores de arroz en cáscara & 16 & $26.7 \%$ \\
\hline Por posibles pérdidas en el pilado (arroz quebrado) & 11 & $18.3 \%$ \\
\hline Por falta de dinero y se necesita mayor tiempo & 17 & $28.3 \%$ \\
\hline Total & 60 & $100.0 \%$ \\
\hline Fuente: Entrevista a productores arroceros, marzo 2019
\end{tabular}

Frente a esta pregunta de vital importancia, el 28.3\% indica por falta de dinero y requieren más tiempo, el $26.7 \%$ manifiesta no hacerlo por desconocimiento al ser sólo productores y no comercializadores, y el $23.3 \%$ por la demora que causa el llevar a pilarlo y venderlo posteriormente. 


\subsection{Identificando rendimientos, ingresos y costos} de producción, pérdidas o ganancias de los productores de arroz en el distrito de Bagua Grande.

Tabla $\mathbf{N}^{\circ} 30$. Rendimiento por hectárea que obtiene durante un ciclo del cultivo en $\mathrm{Kg}$.

\begin{tabular}{c|c|c|}
\multirow{2}{*}{ Kg por Hectárea } & \multicolumn{2}{|c|}{ Total } \\
\cline { 2 - 3 } & Datos & Porcentaje \\
\hline 8,100 & 1 & $1.7 \%$ \\
\hline 8,000 & 7 & $11.7 \%$ \\
\hline 7,900 & 4 & $6.7 \%$ \\
\hline 7,800 & 10 & $16.7 \%$ \\
\hline 7,750 & 1 & $1.7 \%$ \\
\hline 7,700 & 5 & $8.3 \%$ \\
\hline 7,650 & 1 & $1.7 \%$ \\
\hline 7,600 & 14 & $23.3 \%$ \\
\hline 7,500 & 4 & $6.7 \%$ \\
\hline 7,400 & 2 & $3.3 \%$ \\
\hline 7,350 & 1 & $1.7 \%$ \\
\hline 7,300 & 1 & $1.7 \%$ \\
\hline 7,200 & 4 & $6.7 \%$ \\
\hline 7,100 & 1 & $1.7 \%$ \\
\hline 7,072 & 1 & $1.7 \%$ \\
\hline 7,000 & 2 & $3.3 \%$ \\
\hline 6,900 & 0 & $0.0 \%$ \\
\hline 6,800 & 1 & $1.7 \%$ \\
\hline & 60 & $\mathbf{1 0 0 . 0} \%$ \\
\hline Total & 1 & \\
\hline Fyy & 1 & 2019
\end{tabular}

Fuente: Entrevista a productores arroceros, marzo 2019

$7,600 \mathrm{~kg} / \mathrm{ha}$ representan el $23.3 \% ; 7,800 \mathrm{~kg} / \mathrm{ha}$ representan el $16.7 \%$ y el $11.7 \%$ con rendimientos de $8,000 \mathrm{~kg} / \mathrm{ha}$ del total de los productores encuestados. Estos rendimientos superan al obtenido de 7,400 $\mathrm{kg} /$ ha que informó el Ministerio de Agricultura para el año 2016.

Gráfico $\mathbf{N}^{\circ} 01$. Perú, rendimiento promedio de arroz en cáscara por región - año 2016. Rendimiento por hectárea que obtiene durante un ciclo del cultivo en $\mathrm{Kg}$.

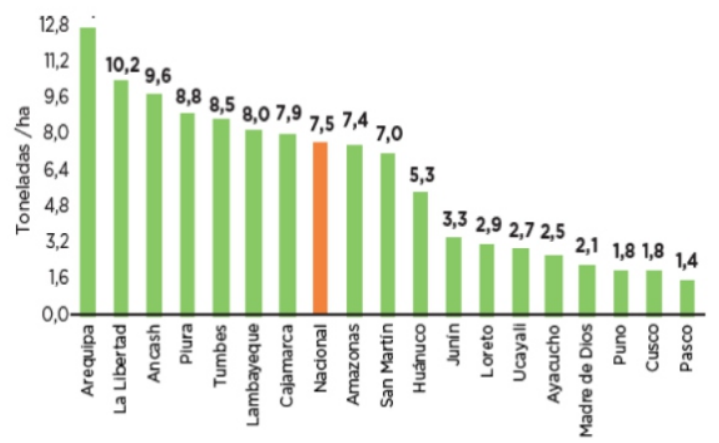

Fuente: MINAGRI-DGESEP

Elaboración: MINAGRI-DGPA
Tabla $\mathrm{N}^{\circ}$ 31. Precio de venta por $\mathrm{Kg}$

\begin{tabular}{|c|c|c|}
\hline \multirow{2}{*}{ Precio por $\mathrm{Kg}$} & \multicolumn{2}{|c|}{ Total } \\
\hline & Datos & Porcentaje \\
\hline S/. 0.91 & 1 & $1.7 \%$ \\
\hline S/. 0.90 & 3 & $5.0 \%$ \\
\hline S/. 0.89 & 4 & $6.7 \%$ \\
\hline S/. 0.88 & 6 & $10.0 \%$ \\
\hline S/. 0.87 & 1 & $1.7 \%$ \\
\hline S/. 0.86 & 1 & $1.7 \%$ \\
\hline S/. 0.85 & 1 & $1.7 \%$ \\
\hline S/. 0.84 & 0 & $0.0 \%$ \\
\hline S/. 0.83 & 1 & $1.7 \%$ \\
\hline S/. 0.82 & 1 & $1.7 \%$ \\
\hline S/. 0.81 & 0 & $0.0 \%$ \\
\hline $\mathrm{S} / .0 .80$ & 2 & $3.3 \%$ \\
\hline S/. 0.79 & 7 & $11.7 \%$ \\
\hline S/. 0.78 & 14 & $23.3 \%$ \\
\hline S/. 0.77 & 7 & $11.7 \%$ \\
\hline S/. 0.76 & 8 & $13.3 \%$ \\
\hline S/. 0.75 & 2 & $3.3 \%$ \\
\hline S/. 0.70 & 1 & $1.7 \%$ \\
\hline & 60 & $100.0 \%$ \\
\hline
\end{tabular}

Fuente: Entrevista a productores arroceros, marzo 2019

$\mathrm{S} / 0.78$ por kg de arroz representan el $23.3 \%$; S/ 0.76 por $\mathrm{kg}$ representan el $13.3 \%$ y S/ 0.77 por $\mathrm{kg}$ del total de los productores encuestados, respectos al precio de arroz vendido en chacra. Estos precios de venta están muy por debajo al obtenido de $\mathrm{S} / 1.03 \mathrm{x} \mathrm{kg}$ que informó el Ministerio de Agricultura para el año 2016.

Tabla $\mathbf{N}^{\circ}$ 32. Costos de producción de arroz por hectárea

\begin{tabular}{|l|c|c|}
\multirow{2}{*}{$\begin{array}{c}\text { Costo producción por } \\
\text { hectárea }\end{array}$} & \multicolumn{2}{|c|}{ Total } \\
\cline { 2 - 3 } & Datos & Porcentaje \\
\hline Entre S/ 3,000 y S/ 3,500 & 0 & $0.0 \%$ \\
\hline Entre S/ 3,501 y S/ 4,000 & 8 & $13.3 \%$ \\
\hline Entre S/ 4,001 y S/ 4,500 & 45 & $75.0 \%$ \\
\hline Entre S/ 4,501 y S/ 5,000 & 7 & $11.7 \%$ \\
\hline Entre S/ 5,001 y S/ 5,500 & 0 & $0.0 \%$ \\
\hline Total & 60 & $100.0 \%$ \\
\hline Fuente: Entrevista a productores arroceros, marzo 2019 \\
\hline
\end{tabular}

El $75 \%$ del total de los productores encuestados identificaron sus costos para producir una hectárea de arroz entre $\mathrm{S} / 4,001$ y S/ 4,500. El 13.3\% gastó entre $\mathrm{S} / 3,501$ y S/ 4,000. Sólo el $11.7 \%$ gastó entre $\mathrm{S} /$ 4,501 y $\mathrm{S} / 5,000$

Tabla $\mathbf{N}^{\circ}$ 33. Balance de ventas por hectárea de arroz.

\begin{tabular}{|c|c|c|}
\hline Ganancias o perdidas & \multicolumn{2}{|c|}{ Total } \\
\cline { 2 - 3 } de producción de arroz & Datos & Porcentaje \\
\hline Entre S/ 501 y S/ 1,000 & 1 & $1.7 \%$ \\
\hline Entre S/ 1,001 y S/ 1,500 & 14 & $23.3 \%$ \\
\hline Entre S/ 1,501 y S/ 2,000 & 27 & $45.0 \%$ \\
\hline Entre S/ 2,001 y S/ 2,500 & 5 & $8.3 \%$ \\
\hline Entre S/ 2,501 y S/ 3,000 & 6 & $10.0 \%$ \\
\hline Entre S/ 3,001 y S/ 3,500 & 7 & $11.7 \%$ \\
\hline Entre S/ 3,501 y S/ 4,000 & 0 & $0.0 \%$ \\
\hline Total & 60 & $100.0 \%$ \\
\hline Fuente: Entrevista a productores arroceros, marzo 2019
\end{tabular}


Una vez identificado el total de sus costos y sus ingresos por la venta de arroz por hectárea a precios en chacra tenemos: El 45\% obtiene entre S/ 1,501 y $\mathrm{S} / 2,000$; el $23.3 \%$ obtiene entre $\mathrm{S} / 1,001$ y S/ 1,500. El $11.7 \%$ obtiene entre S/ 3,001 y S/ 3,500 y el $10 \%$ entre $\mathrm{S} / 2,501$ y $\mathrm{S} / 3,000$.

$\mathrm{Si}$ dividimos estos montos recibidos por la producción de arroz por hectárea vendida a precios de chacra entre 5 meses que dura la campaña, los productores de arroz del distrito de Bagua Grande obtienen en promedio entre $\mathrm{S} / 450$ al mes.

\section{DISCUSIÓN}

Altamirano (2017), en su investigación, realiza un análisis de la rentabilidad a partir del presupuesto de cultivo, ofrece una interesante visión del peso económico que tiene el arroz, las diferencias y similitudes en la tecnología, el uso de los recursos, su productividad, la eficiencia en el uso del agua, la capacidad de absorción de empleo, la capacidad que tiene para cubrir sus costos y de aportar con un margen positivo a la generación de utilidades. La presente investigación también realice un análisis de ingresos y egresos por hectárea de arroz vendida obteniendo ganancias, pero menores a los de las zonas productoras de la costa que tienen mayores rendimientos por hectárea producida al contar con semillas mejoradas.

El aporte que se obtiene de Romero (2017), en su investigación, identifica varias falencias tanto en la parte administrativa como financiera. La empresa no cuenta con un manual de procedimientos, carece de una logística de abastecimiento, no existe un control de calidad para su producción, en la parte financiera no cuenta con un control que le permita evaluar su desempeño económico y verificar si se está cumpliendo con los objetivos planteados; motivos para diseñar varias estrategias que ayuden a resolver los problemas de la empresa y por ende mejorar el desarrollo y rentabilidad de la misma. Con la presente investigación también se identifica ventas y desventajas del sector, planteándose tres estrategias primordiales para solucionar la problemática identificada en los productores de arroz del distrito de Bagua Grande.

El aporte que se obtiene de los autores Aldáz, Sevilla y Ponce (2013), la tesis fue realizado en la provincia de los Ríos Cantón Urdaneta a los pequeños productores de arroz donde previo a un análisis de campo encontraron una serie de falencias donde el agricultor no cuenta con capacitaciones técnicas para la producción de arroz y al mismo tiempo la falta de comercialización directa sin intermediarios para un pago justo de sus cosechas. El análisis de este proyecto pretende lograr que los pequeños productores arroceros se agremien para así juntos obtener beneficios como capacitaciones continuas y la venta directa de sus cosechas sin intermediarios ya que unidos cumplirían el cupo asignado por las almaceneras y mediante esta agremiación lograrían las ganancias esperadas y mejorando su estilo de vida. La presente investigación para el distrito de Bagua Grande coincide con la agremiación de los pequeños productores, pero no se tiene cupos asignados de producción de las almaceneras como lo hay en otros países.

En cuanto a la base teórica sobre: Estrategias para impulsar la alta rentabilidad, sustentado por López (2007), identifica tres estrategias: la de eficiencia, la de vinculación y la de renovación; lo que fueron aplicados en la presente investigación.

\section{CONCLUSIONES}

Del diagnóstico situacional se determinó la capacidad directiva, tecnológica, productiva y comercial de los productores de arroz en el distrito de Bagua Grande, a través de una entrevista en las propias parcelas de los productores de arroz, obteniendo que no están asociados en su gran mayoría, la tecnología es mixta pero obsoleta, la producción se hace sin programaciones efectivas al carecer de recurso hídrico suficiente, y comercialmente no se encuentran asociados y es el intermediario quién compra el arroz en cáscara y lo traslada hacia los molinos de la costa norte del país obteniendo las mayores utilidades de este proceso productivo.

Respecto a los rendimientos, ingresos y costos de producción, pérdidas o ganancias de estos productores arroceros, obtienen ganancias mínimas por campaña de $\mathrm{S} / 350.00$ en promedio por campaña y máximas de $\mathrm{S} / 450.00$ mensuales, perjudicando al pequeño productor menor de 5 hectáreas, principalmente. Pero si identificaron al detalle el total de sus costos y gastos de esta producción.

Al identificar las desventajas del cultivo de arroz en Bagua Grande, en la situación actual estas superan a las ventajas, las que son consideradas un paliativo a mejorar con la propuesta de tres estrategias:

Agrupar a pequeños productores arroceros para hacerlos más competitivos.

- Alianza estratégica en investigación de nueva variedad de arroz con la Universidad Nacional Toribio Rodríguez de Mendoza de Amazonas.

Estudio de pre inversión para la construcción de mini represas de almacenamiento de agua para riego para Bagua Grande, través del gobierno regional Amazonas. 


\section{RECOMENDACIONES}

Evaluar la implementación de esta propuesta a través de la Universidad Nacional Rodríguez de Mendoza de Amazonas, en cuanto a investigación en el sector arrocero, así como el involucramiento del Gobierno Regional Amazonas para la posibilidad de formulación de proyectos de inversión pública a través del Invierte.Pe sobre la construcción de mini represas para almacenar agua para riego.

\section{REFERENCIAS BIBLIOGRÁFICOS}

Aldáz, V.; Sevilla, R. y Ponce, O. (2013). Análisis de la situación socio-económico de los pequeños productores de arroz del cantón Urdaneta y la implementación de un plan de comercialización directa para mejorar su nivel de vida. (Tesis de pregrado). Universidad de Guayaquil, Guayaquil, Ecuador.

Altamirano, E. (2017). Niveles de productividad y rentabilidad del cultivo de arroz en la región norte del Perú: Caso Lambayeque y la Libertad 2000 - 2015. (Tesis de pregrado). Universidad Nacional Agraria La Molina, Lima, Perú.

Angulo, M. y Sarmiento, J. (mayo de 2000). El concepto de rentabilidad en marketing. En M. Angulo (Presidencia), Costos y Contabilidad Directiva. Congreso llevado a cabo en el I Congreso Nacional de Profesores, Santafé de Bogotá, Colombia.

De Bernardi, L. (2016). Perfil del mercado de arroz. $\mathrm{R}$ e c u p e r a d o d e https://www.agroindustria.gob.ar/new/00/pr ogramas/dma/granos/Perfil\%20de $\% 20$ Merc ado\%20de\%20Arroz\%202017.pdf

Dirección Regional Agraria Amazonas (2011). Plan Estratégico Agrario de la región Amazonas 2011-2021. Dirección Regional de Agricultura Amazonas, (5) 29-33.

Hernández, R.; Fernández, C. y Baptista, M. (2010). Metodología de la investigación ( $\left.5^{\mathrm{a}} \mathrm{ed}.\right)$. México: McGraw-Hill.

López, F. (Ed.). (2007). Estrategias para alcanzar y mantener la alta rentabilidad. Vigo, España: Editorial Obradoiro gráfico.

Ministerio de Agricultura y Riego. (2017). Análisis económico 2017. Informe del arroz. $\mathrm{R}$ e c u p e r a d o

d e http://www.minagri.gob.pe/portal/analisiseconomico/analisis-2017
Ministerio de Agricultura y Riego. (2019). Observatorio de Commodities Arroz 2019. Informe del arroz. Boletín de publicación trimestral enero-marzo Dirección General de Políticas Agrarias, (11) 1-11.

Romero, N. (2017). Diseño de estrategias para mejorar la rentabilidad de la empresa Produarroz S.A. (Tesis de pregrado). Universidad de Guayaquil, Guayaquil, Ecuador. 